\title{
POTENTIAL BIOMARKERS FROM POSITIVE DEFINITE 4TH ORDER TENSORS IN HARDI
}

\author{
Sumit Kaushik, Jan Kybic \\ Faculty of Electrical Engineering \\ Czech Technical University in Prague \\ Czech Republic
}

\begin{abstract}
In this paper, we provide a framework to evaluate new scalar quantities for higher order tensors (HOT) appearing in high angular resolution diffusion imaging (HARDI). These can potentially serve as biomarkers. It involves flattening of HOTs and extraction of the diagonal D-components. Experiments performed in the 4 th order case reveal that D-components encode geometric information unlike the isometric 6D 2nd order Voigt form. The existing invariants obtained from the Voigt form are considered for comparison. We also notice that Dcomponents can be useful in segmentation of white matter structures in crossing regions and classification. Results on phantom and the synthetic dataset support the conclusions.
\end{abstract}

Index Terms - HARDI, biomarker, 4th order tensor, Dcomponent

\section{INTRODUCTION}

Diffusion MRI (dMRI) is a powerful tool in the study of microstructures in human brain non-invasively. Based upon dMRI principle, diffusion tensor imaging (DTI) model was introduced by Basser and others [1], [2] and its importance as a clinical standard grows. The eigenvalues of the 2 nd order DTI tensors remain invariant under 3D rotations. Some of the scalars obtained as from function of these eigenvalues are: mean diffusivity (MD), fractional anisotropy (FA), radial diffusivity (RD) etc. These scalars serve as the biomarkers which are helpful in discerning unhealthy tissues from healthy ones. This change occurs due to disorganization of tissue structure due to present anomaly. The DTI modality looses its efficiency if the diffusion of water molecules is not Gaussian, which happens e.g. for voxels including crossing or merging fibers. For such situations, we may increase the number of the measured diffusion gradient directions [3] and approximate the diffusion by higher order tensors. This acquisition protocol is known as high angular diffusion imaging (HARDI). There are some works which considered rotational invariance property to obtain scalars in higher order tensors (HOT). These scalars are shown to serve as potential biomarkers and are considered better than those derived from 2nd order tensors. Ghosh et al proposed such invariants of

\author{
Avinash Bansal, Temesgen Bihonegn, Jan Slovak
}

\author{
Department of Mathematics and Statistics \\ Masaryk University \\ Brno, Czech Republic
}

4th order tensor [4]. They are obtained from the characteristic polynomial of the Voigt form, a $6 \times 6$ isometric representation of the 4th order tensors. Their eigenvalues are also known as Kelvin eigenvalues and they are invariant under 6D rotation group $S O(6)$ instead of the real 3D rotations of interest. Further works deal with the complete set of invariants under 3D rotations [5].

The biological and clinical significance of these HOT rotation invariant scalars is still largely a matter of research. In this paper, we present an approach which extracts scalar measures of positive definite higher order tensors using the diagonal component (D) approach. It is observed that the three $3 \times 3$ (blocks) diagonal components in case of 3D flattened 4 th order tensor carry useful geometric information. The scalar obtained by combining these components is shown to be robust under rotations than those aforementioned. The principal eigenvalues of the these components reflect the number of fibers at a voxel level. These scalars can serve as potential biomarkers. We have shown they are also effective in segmenting white matter fibers in heterogeneous region and classification of tissues with respect to underlying number of fibers. The approach is extendable to HOT of any order. We discuss experiments on phantom and synthetic dataset.

\section{THEORY}

\subsection{Diffusion Model}

The generalized Stejskal-Tanner equation which is a mono exponential model of the diffusion of water molecules in biological tissues. The attenuated signal corresponding to a gradient pulse, with the diffusion weighting coefficient $b$, is $S(\mathbf{g})=S_{0} \exp (-b D(\mathbf{g}))$, where

$$
D(\mathbf{g})=\sum_{j_{1}=1}^{3} \sum_{j_{2}=1}^{3} \ldots \sum_{j_{n}=1}^{3} D_{j_{1} j_{2} \ldots j_{n}} g_{j_{1}} g_{j_{2}} \ldots g_{j_{n}}
$$

and $g_{k}$ is the $k$ th component of the magnetic gradient vector with $|\mathbf{g}|=1$. The number of independent coefficients for $\ell$ th order symmetric tensors is $N_{\ell}=\frac{1}{2}(\ell+1)(\ell+2)$. Thus, for 4 th order symmetric tensors, $3^{4}=81$ coefficients of general tensors reduce to 15 . 


\subsection{Related work}

A 3D 4th order tensor can be represented in the so called Voigt notation by $6 \times 6$ matrix $M$, [6]. The eigenvalues $\lambda_{i}$, $i=1, \ldots, 6$, of this matrix are called the Kelvin eigenvalues, and they give rise to the basic invariants $S 4_{r}$, obtained as the six traces of the powers $M^{r}, r=1, \ldots, 6$. Further 6 invariants $J 4_{r}$, called the principal ones, appear as the coefficients of the characteristic polynomial $\operatorname{det}(M-\lambda I)$. In fact $J_{41}=S_{41}$. As it is well known, such scalars are independent of the choice of basis and thus also rotation invariant. Unfortunately the invariance is with respect to the 6-dimensional rotations.

Since the Voigt form of the tensor is a positively definite symmetric matrix, we may write the explicit formulas for the above invariants in terms of the Kelvin eigenvalues, e.g.

$$
\begin{aligned}
S 4_{1} & =J 4_{1}=\operatorname{tr}(M)=\lambda_{1}+\lambda_{2}+\lambda_{3}+\lambda_{4}+\lambda_{5}+\lambda_{6}, \\
S 4_{2} & =\operatorname{tr}\left(\mathrm{M}^{2}\right)=\lambda_{1}^{2}+\lambda_{2}^{2}+\lambda_{3}^{2}+\lambda_{4}^{2}+\lambda_{5}^{2}+\lambda_{6}^{2}, \\
J 4_{3} & =\lambda_{1} \lambda_{2} \lambda_{3}+\lambda_{1} \lambda_{2} \lambda_{4}+\lambda_{1} \lambda_{2} \lambda_{5}+\lambda_{1} \lambda_{2} \lambda_{6} \\
& +\lambda_{1} \lambda_{3} \lambda_{4}+\lambda_{1} \lambda_{3} \lambda_{5}+\lambda_{1} \lambda_{3} \lambda_{6}+\lambda_{1} \lambda_{4} \lambda_{5}+\lambda_{1} \lambda_{4} \lambda_{6} \\
& +\lambda_{1} \lambda_{5} \lambda_{6}+\lambda_{2} \lambda_{3} \lambda_{4}+\lambda_{2} \lambda_{3} \lambda_{5}+\lambda_{2} \lambda_{3} \lambda_{6}+\lambda_{2} \lambda_{4} \lambda_{5} \\
& +\lambda_{2} \lambda_{4} \lambda_{6}+\lambda_{2} \lambda_{5} \lambda_{6}+\lambda_{3} \lambda_{4} \lambda_{5}+\lambda_{3} \lambda_{4} \lambda_{6}+\lambda_{3} \lambda_{5} \lambda_{6} \\
& +\lambda_{4} \lambda_{5} \lambda_{6}, \\
J 4_{6} & =\operatorname{det}(M)=\lambda_{1} \lambda_{2} \lambda_{3} \lambda_{4} \lambda_{5} \lambda_{6} .
\end{aligned}
$$

For the complete set of equations reader is referred to [6] and [4].

\subsection{The proposed approach}

Tensors are multi-linear maps whose components transform in a co/contra-variant manner. However, in presence of fixed scalar product at each point of space, i.e., for the Cartesian tensors these transformations are indistinguishable. All Cartesian tensors can be represented in isometric vector forms, but in general geometric information is lost.

We propose another approach. Any 4th order tensor can be interpreted as a 9x9 matrix

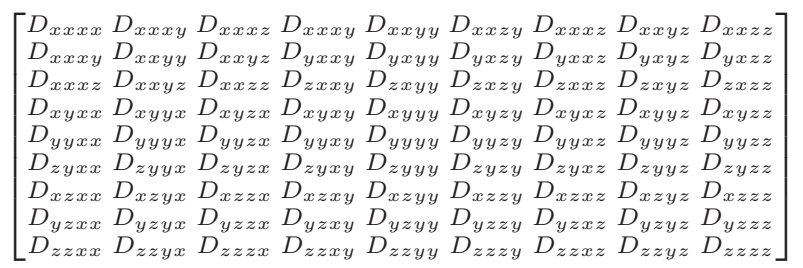

which can be understood as a $3 \times 3$ matrix whose elements are again $3 \times 3$ matrices (here $D$ are the coefficients appearing in the Stejskal-Tanner equation (1)). We call them Dcomponents (DC). The first such diagonal element in the fourth-order tensor is

$$
T_{x x}^{(2)}=\left(\begin{array}{lll}
D_{x x(x x)} & D_{x x(x y)} & D_{x x(x z)} \\
D_{x x(x y)} & D_{x x(y y)} & D_{x x(y z)} \\
D_{x x(x z)} & D_{x x(y z)} & D_{x x(z z)}
\end{array}\right)
$$

In general, every $n$th order tensor $T^{(n)}$ can be expressed as a $3 \times 3$ matrix of $(n-2)$-nd order tensors. For instance, there are three such diagonal $3 \times 3$ components for 4 th order tensors, while for the 6th order, the three components further contain nine such diagonal $3 \times 3$ components in total.

Due to the physical nature of diffusion process, all tensors $T^{(n)}$ must be positive semi-definite (PSD) which is ensured by methods introduced in [7] and [8] while fitting the signal. As discussed in [9], the semi-definiteness can be inherited by all the second order diagonal components. These form a subset of all possible principal minors. The spectral decomposition of the tensor in equation 2 is given by $T_{x x}^{(2)} V_{x x}=\Lambda_{x x} V_{x x}$. Similarly for other two components as $T_{y y}^{(2)} V_{y y}=\Lambda_{y y} V_{y y}$ and $T_{z z}^{(2)} V_{z z}=\Lambda_{z z} V_{z z}$

Here, $\Lambda$ 's are diagonal matrices and $V$ matrices hold eigenvectors as their columns. The $\lambda_{x x}, \lambda_{y y}$ and $\lambda_{z z}$ are maximum eigenvalues corresponding to $\Lambda_{x x}, \Lambda_{y y}$ and $\Lambda_{z z}$ respectively.

While the eigenvectors of the D-components can be used to extract the direction of maxima of orientation distribution function (ODF), we focus here on the eigenvalues which can provide new biomarkers in a straightforward way. The normalized vector at each voxel is given by

$v_{n}=v /\left(\lambda_{x x}+\lambda_{y y}+\lambda_{z z}\right)$. Here, $v=\left(\lambda_{x x}, \lambda_{y y}, \lambda_{z z}\right)$ is computed from the maximal eigenvalues of the D-components. These elements of $v_{n}$ can be considered as probabilities of presence of corresponding fibers.

The eigenvalues of D-components can be combined to give scalars which are robust under rotations. For instance, we evaluated fractional anisotropy (FA) of sum of the Dcomponents in experiments. For 4th order case, these components provide 3 -fold information per voxel each corresponding to the individual D component. This has biologically and clinically more relevance than the invariants obtained from the Voigt 6x6 representation. Also, the method can be easily extended to any even order tensor. It should be noted that the eigenvalues of D-components are different from those obtained from spectral decomposition of 9x9 or the $6 \times 6$ representations.

Eigen decomposition of $3 \times 3$ SPD blocks is faster than for $6 \times 6$ or $9 \times 9$ matrices and there is available a rich class of methods to obtain biomarkers due to active research on DTI modality in the past years. These are all applicable to the Dcomponents due to the SPD property mentioned above.

\section{EXPERIMENTS AND RESULTS}

We simulated synthetic images (64 gradient direction with $\mathrm{b}=1500 \mathrm{~s} / \mathrm{mm}^{2}$ ) using an adaptive kernel method [10]. 

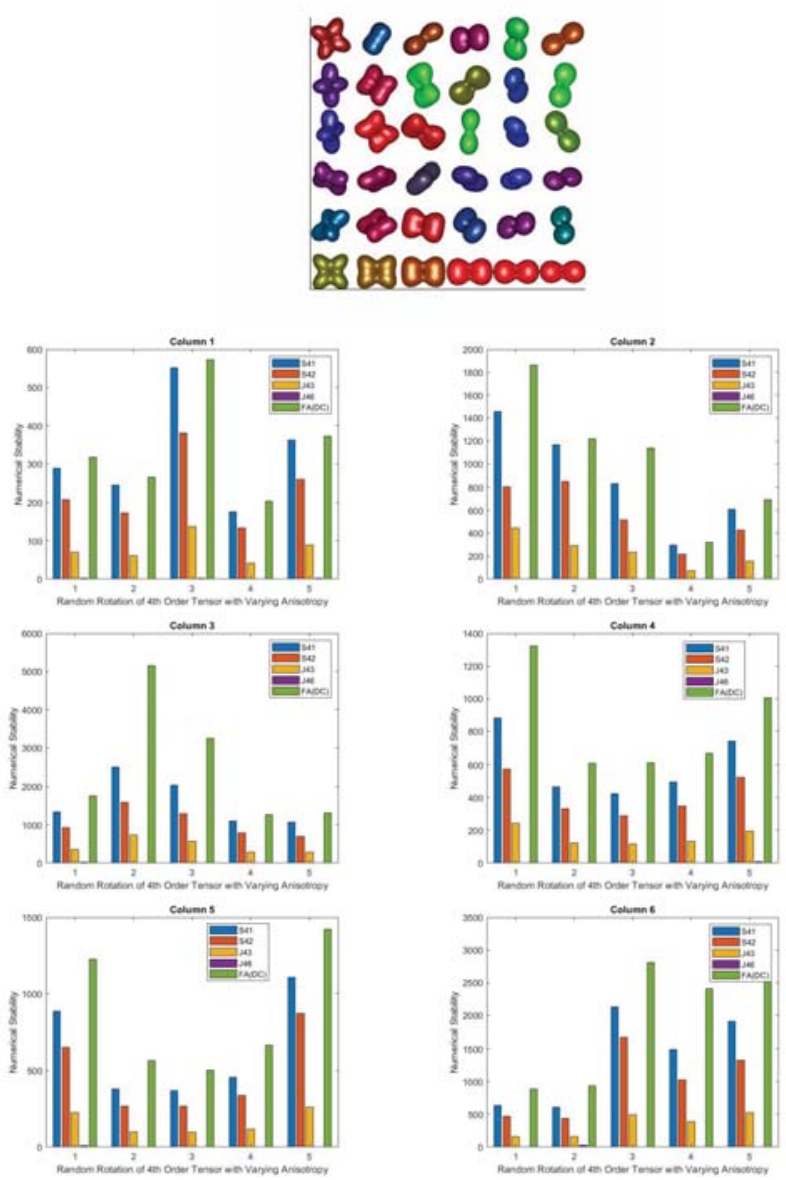

Fig. 1. Comparison of the numerical stability with respect to random rotations of the Kelvin based invariants and the FA of the D-components (the green bars). The higher bar indicate higher stability.

For 6 different 4 th order tensors $T_{1}$ through $T_{6}$, constructed with decreasing angle between two fibers, we randomly chose five 3D rotations of each of them, i.e., we consider the corresponding 5 configurations $T_{i j}(i=1, \ldots, 6$ and $j=1, \ldots, 5)$. The numerical stability with respect to random rotations is defined as $\frac{\left|S\left(T_{i}\right)\right|}{\left|S\left(T_{i}\right)-S\left(T_{i j}\right)\right|}$, where $S(T)$ stands for the scalar measure for tensor $T$. We include the FA for sum of diagonal components, and a set of scalars obtained from the Voigt form: $S 4_{1}=J 4_{1}, S 4_{2}, J 4_{3}$ and $J 4_{6}$.

Figure 1 indicates the higher numerical stability of the scalar based on the D-components, in comparison to those based on Kelvin eigenvalues.

Figure 2 depicts two perpendicular crossing fibers and the various scalar images. The scalar images corresponding to $\lambda_{x x}, \lambda_{y y}, \lambda_{z z}$ eigenvalues are also shown in third row. The red, green and blue colors in the vector image are represented by $v_{n}(1), v_{n}(2)$ and $v_{n}(3)$ respectively. The normalized vector discussed above is scaled to $v_{n} / \max \left(v_{n}\right)$. Then, we select $>0.5$ to threshold individual terms. This provides the number
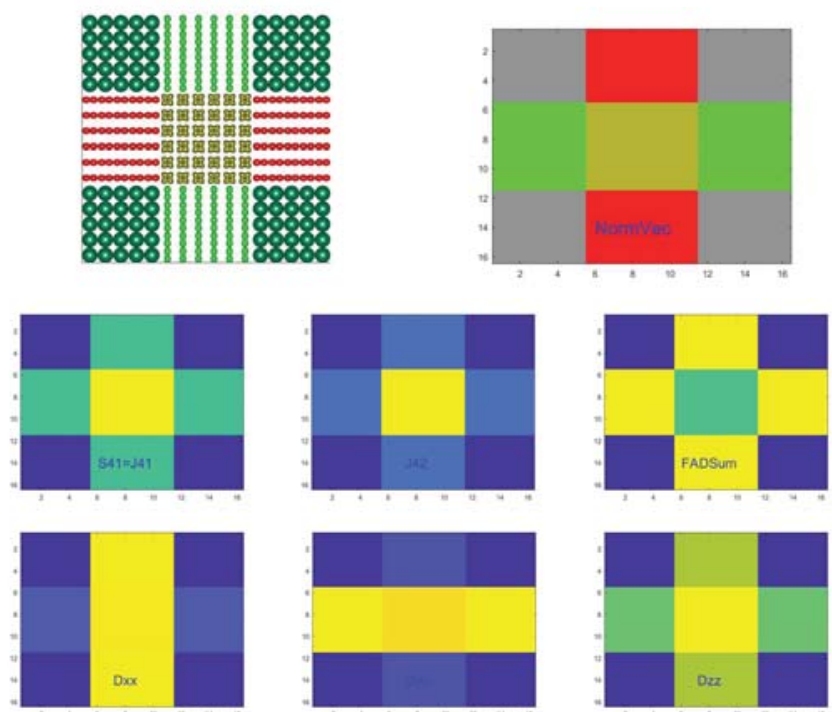

Fig. 2. First row: Synthetic 4th order tensor image of two crossing fibers and the RGB image of the normalized vector $v_{n}$. Second row: two examples of the Kelvin eigenvalues based invariants and the FA of the sum of D-components (using the default Matlab color scale) Third row: the separate D-components are able to depict the two fibers individually and the intersection regions (in different color code)

Table 1. The mean errors for 1000 random rotations each

\begin{tabular}{l|c|c|c|c|c|} 
S41 & S42 & S43 & S44 & S45 & S46 \\
\hline 0.0023 & 0.0033 & 0.0047 & 0.0062 & 0.0078 & 0.0094 \\
\hline \hline J42 & J43 & J44 & J45 & J46 & FA-DC \\
\hline 0.0052 & 0.0093 & 0.0162 & 0.0401 & 0.2963 & 0.002
\end{tabular}

of fibers in a voxel. This is used for classification of voxels according to underlying fibers present/absent. For instance, in figure 2, the vector image contains two single fiber regions shown in green and red, background has isotropic tensors indicating no fibers whereas the brown color (red + green) represents the region where two fibers are intersecting. We perform 1000 random rotations of a fixed 4th order tensor and computed the relative errors for each of these scalars. Table 1 shows the scalar due to DC has least error.

Figure 3 provides results for the phantom used in [11]. There, the puree of asparagus in equal amounts is used to simulate three orthogonal fiber directions. The size of phantom is $64 \times 64 \times 6$. The results are shown for the 4th slice. Aside the original structural image, the picture shows the normalized vector $v_{n}$ image as mentioned above. It can be seen that three directions are very well captured by the D-components individually. In second row, the first image shows tensor field for the two curved intersecting fibers. second image shows classification of the 3 regions. The red indicates region having 1 

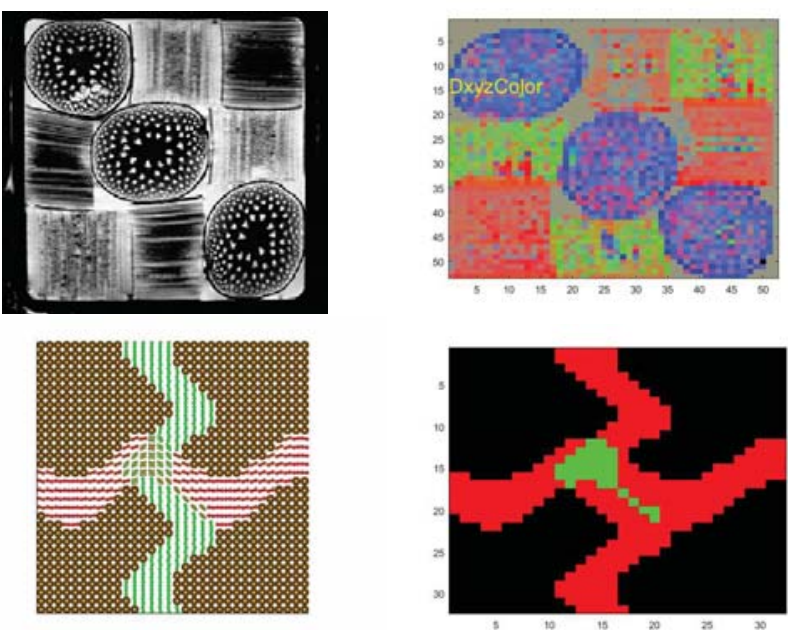

Fig. 3. First row: structural image of the phantom, followed by the normalized vector image. Second row: Two closely merging fibers, the normalized vector image segregating the regions according to number of fibers

fiber, black for isotropic background and green represents the presence of two fibers. It should be noted that the intersecting region is classified accurately even in the part where two curves are barely touching.

\section{CONCLUSION}

We showed how to compute D-components from HOTs which carry geometric information whereas the 6x6 Voigt form fails in this respect.The method applies for tensors of all even orders. We showed that the scalar quantities based on the Dcomponents are robust under rotation of tensors with varying anisotropies. Diverse experiments on phantom and synthetic images, confirmed that the individual D-components and their combinations capture the relevant information and this simple framework provides a promising way to look for HARDI scalars providing classification of single and multi-fiber regions.

\section{Compliance with Ethical Standards}

This is a numerical simulation study for which no ethical approval was required.

\section{Acknowledgments}

The authors from CTU, Prague acknowledges the support by OpVVV project CZ.02.1.01/0.0/0.0/16 019/0000765 Research Center for Informatics. The first two authors from MU are supported by the grant MUNI/A/0885/2019, Jan Slovák acknowledges support from the GACR, grant Nr. GA2011473 S.

\section{REFERENCES}

[1] Peter J Basser, James Mattiello, and Denis LeBihan. Estimation of the effective self-diffusion tensor from the NMR spin echo. Journal of Magnetic Resonance, Series $B, 103(3): 247-254,1994$.

[2] Peter J, James Mattiello, and Denis LeBihan. MR diffusion tensor spectroscopy and imaging. Biophysical journal, 66(1):259-267, 1994.

[3] David Solomon Tuch et al. Diffusion MRI of complex tissue structure. PhD thesis, Massachusetts Institute of Technology, 2002.

[4] Aurobrata Ghosh, Théo Papadopoulo, and Rachid Deriche. Biomarkers for HARDI: 2nd \& 4th order tensor invariants. In 2012 9th IEEE International Symposium on Biomedical Imaging (ISBI), pages 26-29. IEEE, 2012.

[5] Aurobrata Ghosh, Théodore Papadopoulo, and Rachid Deriche. Generalized invariants of a 4 th order tensor: Building blocks for new biomarkers in dMRI. In Proceedings of the Computation Diffusion MRI Workshop at the MICCAI Conference, 2012.

[6] Peter J Basser and Sinisa Pajevic. Spectral decomposition of a 4th-order covariance tensor: Applications to diffusion tensor MRI. Signal Processing, 87(2):220236, 2007.

[7] Angelos Barmpoutis, Min Sig Hwang, Dena Howland, John R Forder, and Baba C Vemuri. Regularized positive-definite fourth order tensor field estimation from DW-MRI. NeuroImage, 45(1):S153-S162, 2009.

[8] Angelos Barmpoutis and Baba C Vemuri. A unified framework for estimating diffusion tensors of any order with symmetric positive-definite constraints. In 2010 IEEE international symposium on biomedical imaging: from nano to macro, pages 1385-1388. IEEE, 2010.

[9] Sumit Kaushik and Jan Slovák. HARDI segmentation via fourth-order tensors and anisotropy preserving similarity measures. Journal of Mathematical Imaging and Vision, 61:1221 - 1234, 2019.

[10] Bing Jian Barmpoutis and Baba C. Vemuri. Adaptive kernels for multi-fiber reconstruction. Information processing in medical imaging : proceedings of the conference, 21:338-49, 2009.

[11] Brian Hansen and Sune Nørhøj Jespersen. Kurtosis fractional anisotropy, its contrast and estimation by proxy. Scientific reports, 6:23999, 2016. 